Abstract

2 The rapid growth in forms of technology used within society presents the field of sport and

3 exercise psychology with numerous opportunities and considerations. Nevertheless, there

4 currently exists a scarcity of knowledge or research for Sport Psychology Professionals (SPPs) to

5 draw from when seeking to use technology and media in their service delivery. This article

6 presents a commentary on the perceived opportunities and considerations drawing primarily from

7 the online counseling literature and we offer recommendations for good practice for SPPs

8 utilizing technology and media in service delivery.

Keywords: Sport Psychology Professionals, Online service delivery, Computer-mediated

10 communication, Technology.

11

12

13

14

15 Opportunities and considerations of new media and technology in sport psychology service delivery

16

17

David Price, Chris Wagstaff \& Richard Thelwell

18

19

20

21

22

23

24 


\section{ONLINE SERVICE DELIVERY}

The rapid growth and expansion in technological advancements (e.g., video

29 conferencing, mobile applications, social media) has recently received scholarly attention in the

30 field of sport and exercise psychology (Bird \& Harris, 2019; Cotterill \& Symes, 2014; Cottrell et.

31 al, 2019). This growing interest and upsurge in the use of technology in the provision of sport

32 psychology, has been further accentuated by the COVID-19 pandemic. As such, these

33 advancements in technology have benefitted the profession by offering Sport Psychology

34 Professionals (SPPs) new and innovative ways of working with clients. For example, SPPs have a

35 range of online platforms (e.g., Skype, Zoom, Facetime, Google Hangouts, Cisco WebEx,

36 Microsoft Teams) to utilize when working with individuals online. Further, some of these online

37 platforms also offer additional functions (e.g., breakout rooms, screenshare), which enable

38 practitioners to work more creatively with teams online.

Traditionally, SPPs have employed conventional face-to-face consultancy methods when

40 delivering the provision of sport psychology (Fletcher \& Wagstaff, 2009). While these

41 approaches continue to have substantial merit, the current landscape in which SPPs operate in

42 continues to evolve (Sly et al., 2020). Therefore, SPPs are required to consider new ways of

43 working to ensure effective sport psychology service delivery. Given the atypical conditions of

44 the professional environment (e.g., time, location, long working hours), one way in which the

45 provision of sport psychology has evolved to meet these demands, is through the emergence of

46 working in geographically dispersed teams. Geographically dispersed teams are defined as

47 groups that are separated by time and/or distance and must undertake work that includes

48 technology, teamwork and communications (Sessa et al., 1999). This definition aligns with the

49 current landscape in which SPPs operate in, given that it is not uncommon for SPPs to work with 50 clients across different time zones and locations. 
In addition to the influence of the professional environment in which SPPs operate in,

6

clients seeking to use the services of SPPs have also been influenced by technological advancements. For example, clients may now own numerous electronic devices (personal computers, tablets, cellular/mobile telephones), that allow them to interact with SPPs in a variety of capacities (e.g., e-mail, phone call, video conferencing; cf. Blind Manuscript). In light of this, SPPs are challenged to respond to these new forms of communication while being mindful of the impact of these changes to their effectiveness and manage the expectations and needs of their clients as part of a continued development and evolution of their practice (Cottrell et. al, 2019; Quartiroli et al. 2019). Indeed, these technological advancements offer a number of opportunities and considerations for SPPs operating in the field. The aim of this article is two-fold. First, we aim to highlight the opportunities and considerations of using technology in online service delivery regarding the working alliance, accessibility, anonymity and disinhibition, time delays, absence of verbal and nonverbal cues, and technological issues. Second, we aim to provide good practice recommendations for SPPs seeking to use technological-based consultancy approaches.

\section{Opportunities and Considerations}

While traditional face-to-face consultancy methods continue to be a popular method of sport psychology service delivery, technology offers new ways for SPPs to work with clients. Despite the growing interest into the role media and technology play in the provision of sports psychology (Bird \& Harris, 2019; Cottrell et al., 2019), there remains a paucity of research available to inform SPPs practice. Nevertheless, there exists a body of online counseling literature (Richards \& Viganó, 2013; Harris \& Birnbaum, 2015) that may provide a useful resource for SPPs to draw from when attempting to develop effective online services.

Online counseling has been defined as "the delivery of therapeutic interventions in cyberspace where the communication between a trained professional counsellor and client(s) is facilitated using computer-mediated communication (CMC) technologies, provided as a standalone service or as an adjunct to other therapeutic interventions" (Richards \& Viganó, 2012, p. 
699). This form of service provision is unfortunately often used interchangeably with e-therapy, e-counseling or cybertherapy, which results in conceptual confusion on how it is defined. While a range of $\mathrm{CMC}$ technologies are available, these can be broadly categorized as being either asynchronous or synchronous. Asynchronous communication (e.g., emails) means that the client's and practitioners communication do not occur simultaneously (Barak \& Grohol, 2011) whereas, synchronous communication (e.g., video conferencing) means that the sessions occur in real time (Elleven \& Allen, 2004). Additionally, these types of communication modalities can either be conducted singularly or in conjunction with one another. To illustrate, a practitioner may conduct a video-based consultation with a client (synchronous) and then follow-up after the session with an email (asynchronous). As such, asynchronous and synchronous communication both present opportunities and considerations for practitioners when using $\mathrm{CMC}$ technologies. In drawing on the most prominent of these opportunities and considerations, we pay specific attention to: working alliance; accessibility; anonymity and disinhibition; time delays; absence of verbal and nonverbal cues; and, technological issues.

\section{Working Alliance}

The working alliance is a term used synonymously with the therapeutic relationship or client-practitioner relationship, and refers to a negotiated, collaborative feature of the helping relationship (Bordin, 1979). It is recognized by researchers (Orlinsky et al., 2004), that the working alliance is an integral component in the successful outcome of any intervention. As such, an absence of the working alliance or a failure to develop one, is likely to undermine the effectiveness of an intervention (Gelso \& Hayes, 1998). Although, a plethora of research exists examining the role the working alliance plays in traditional face-to-face counseling (Orlinsky et al., 2004), the extant literature on the working alliance in online service delivery remains scarce.

Consequently, this presents a significant challenge for practitioners in cultivating equally meaning relationships with clients when employing $\mathrm{CMC}$ technologies and ultimately promoting effective service delivery (Richards \& Viganó, 2013). 
In an early review of the literature regarding the working alliance in online counseling,

104 Mallen et al. (2005) reported equivocal findings when comparing the strength of the working

105 alliance in face-to-face counseling and online counseling. Since the review of literature, a

106 growing body of research (Leibert et al., 2006; Buffini \& Gordon, 2015) has been conducted,

107 which has revealed more positive findings to support the notion that developing a working

108 alliance in online service delivery is feasible. For instance, Leibert et al. (2006) found the

109 working alliance to be a predictor of client satisfaction in e-therapy. Nevertheless, the strength of

110 the working alliance and client satisfaction were reported higher in face-to-face counseling than

111 clients who received e-therapy. Similarly, Buffini and Gordon (2015) reported a significant

112 positive relationship between the strength of the working alliance and client satisfaction when

113 using synchronous instant messaging services for crisis interventions. Further, when comparing

114 these findings to previous research conducted in offline settings, participants scored significantly

115 lower on both working alliance and client satisfaction ratings.

More recently, qualitative researchers (Barrazzone et al., 2012; Cipolletta et al., 2018)

117 have attempted to explore the relational features underpinning the working alliance in online

118 service delivery. Barrazzone and colleagues (2012) conducted a qualitative inquiry into whether

119 relational features in establishing, developing and maintaining a working alliance, could be

120 translated into a computerized CBT program. These findings can be interpreted to indicate that

121 relational features in establishing (e.g., empathy, warmth, unconditional acceptance), developing

122 (e.g., feedback, developing a secure base) and maintaining (e.g., flexibility, responsiveness) were

123 emulated in a computerized CBT program.

In sum, while the body of literature regarding the working alliance in online service

125 delivery is in its infancy, some important considerations can be drawn to inform SPPs practice.

126 Most notably, it is possible that the relational imperatives critical to establishing, developing and

127 maintaining a working alliance within face-to-face counseling can be achieved via online service

128 delivery settings yet, it is important for practitioners to be cognizant of the limitations of 
129 developing an online working alliance. Put simply, it is still possible for SPPs to foster a working

130 alliance online with their client in order to facilitate behavior change.

\section{Accessibility}

One of the most frequently cited advantages of using technology in online service

delivery is the increased accessibility for both clients and practitioners. Given the unique context

in which SPPs operate in (e.g., clientele, atypical working settings), technology provides greater access to working with clients online and can help reduce barriers that are often associated with

136 traditional face-to-face counseling services (e.g., geographical constraints, limited hours, mobility

137 challenges) (Barnett, 2005). As a result, technology provides clients in remote or underserviced

138 areas greater flexibility and accessibility in engaging in the provision of psychological support

139 (Riemer-Reiss, 2000). In addition, clients seeking to engage in face-to-face counseling services

140 may feel stigmatized by the service delivery process and experience feelings of shame (Rochlen

141 et al., 2004). Online service delivery lends itself as a viable alternative that may be more

142 accessible to such individuals, as it has the potential to reduce the stigma attached with seeking

143 help (Rochlen et al., 2004) and can also enhance positive help seeking attitudes (Chang, 2005).

144 While the use of online service delivery opens up new opportunities through increased

145 accessibility, the use of technology may also pose accessibility challenges to both clients and

146 practitioners. Individuals who do not have access to technology or possess the requisite skills and

147 knowledge to effectively utilize such services, may perceive online service delivery as

148 inaccessible (Riemer-Reiss, 2000; Elleven \& Allen, 2004). Indeed, limited technological literacy

149 is a challenge to online service delivery therefore, practitioners must take precautions not to

150 alienate and/or isolate the client's technological literacy ability (Elleven \& Allen, 2004).

151 Interestingly, the increased accessibility afforded through using technology in online service

152 delivery may also pose ethical concerns regarding communication boundaries, as SPPs may be

153 perceived by client's as being accessible at all times. The potential risk of blurred communication

154 boundaries is an important consideration for SPPs when using technology in the online service 
155 delivery process, which emphasizes the importance for SPPs to manage client's expectations on

156 availability by negotiating and establishing a set of clear boundaries at the outset of consultancy.

\section{Anonymity and Disinhibition}

It is often reported in the online counseling literature (Richards \& Viganó, 2013; Harris \&

159 Birnbaum, 2015) that clients enjoy the natural sense of anonymity provided by the online

160 environment. Traditionally, clients who sought online service delivery were able to engage with

161 such services without disclosing identifiable information (Harris \& Birnbaum, 2015).

162 Nevertheless, the changes to professional guidelines and ethical standards has meant that the

163 process of how clients are recruited, identified and assessed have changed (Richards \& Viganó,

164 2013). In particular, these changes to professional guidelines and ethical standards have arisen to

165 consider the risks associated when working with children and young people online. For example,

166 assessing whether the client is Gillick competent or to ensure a required level of confidentiality is

167 achieved by knowing who else is in the room or nearby during consultancy.

Although changes to the professional guidelines and ethical standards of online service

169 delivery have removed a full sense of anonymity for clients, it is still possible for clients to

170 experience, to some extent, a natural sense of anonymity. In particular, perceptions of anonymity

171 when engaging in online service delivery have been suggested to facilitate psychological safety,

172 disinhibition and increased self-disclosure (Suler, 2010). While traditional counseling services

173 often have social stigmas attached (e.g., gender, physical appearance) when seeking professional

174 support (Efstathiou, 2009), the perceived anonymity offered through online service delivery has

175 the potential to reduce such stigmas and the associated anxieties. In turn, this may result in

176 increased disinhibition and accelerate the rate at which clients disclose information due to the

177 lack of nonverbal cues available (Rochlen et al., 2004).

178 In sum, online service delivery may increase feelings of safety, diminish a client's sense

179 of vulnerability and play a facilitative role in disclosure, and assisting the working alliance (cf.

180 Harris \& Birnbaum, 2015). Therefore, SPPs should carefully integrate technology in the service 
181 delivery process to create environments that are psychologically safe and provide a sense of

182 perceived anonymity, which will increase disinhibition and result in more effective practice.

\section{Time Delays}

An important distinction made between CMC technologies is whether they can be

categorized as asynchronous or synchronous. Synchronous communication allows clients and communication does not occur in real time and has a natural time delay built into the service

188 delivery process (e.g., emails). Any communication time delay has the potential to lead to anxiety being experienced by either the client or practitioner following a perceived or unexplained delay in response (Richards \& Viganó, 2013). Further, the ambiguity that can be experienced when a

191 response is delayed can cause a blank screen, whereby one projects their own expectations, 192 emotions and anxieties (Suler, 2004). While therapeutic interventions delivered through 193 asynchronous communication will differ considerably to those delivered through traditional 194 approaches, the time delay may mean that what can be achieved in one face-to-face session could 195 potentially take several days or weeks in asynchronous online service delivery (Barnett, 2005). Nevertheless, the use of asynchronous communication may serve as an adaptive function

197 especially, given the atypical working environment that SPPs operate in. For example,

198 asynchronous communication may be important when consulting with clients competing in

199 different time zones or employed as an alternative to synchronous communication to promote 200 professional quality of life when experiencing 'compassion fatigue' (Quartiroli et al., 2019). 201 Asynchronous communication also has the potential to facilitate the development of a zone of 202 reflection, whereby clients and practitioners have the opportunity to take their time to engage in 203 deeper reflection before responding to messages (Richards \& Viganó, 2013). As such, this level 204 of reflection might enable clients to process experiences, emotions, reduce impulsivity and 205 enhance self-awareness and self-expression (Hanley, 2009). 
It is well-recognized by consumers of sport psychology (e.g., athletes, coaches, parents, gatekeepers) that interpersonal skills are desirable characteristics of SPPs (Thelwell et al., 2018;

209 Woolway \& Harwood, 2019; Woolway \& Harwood, 2020). In particular, verbal (e.g., tone, 210 speech) and nonverbal cues (e.g., body language) are of critical importance to practitioners in

211 gaining an understanding and insight into the client's thoughts, feelings and behaviors (Harris \&

212 Birnhaum, 2015). Unlike traditional face-to-face counseling that typically offers able-bodied

213 observations of verbal and nonverbal cues, practitioners are less able to observe such cues in

214 online service delivery. This absence of verbal and nonverbal cues poses communication

215 challenges for both the client and practitioner which may mean that information could be 216 misinterpreted or misunderstood. Consequently, this may negatively affect the working alliance

217 and reduce the effectiveness of service delivery (Harris \& Birnhaum, 2015; Bird \& Harris, 2019). While the absence of verbal and nonverbal cues is recognized as a limitation of online 219 service delivery, scholars (e.g., Suler, 2004; Hanley, 2009) argue that the absence of such cues 220 could benefit the service delivery process. To elaborate, clients have often reported that the 221 absence of nonverbal cues can help alleviate any concerns they hold, with regards to the 222 practitioner's reactions when disclosing personal information (Richards \& Viganó, 2013). In turn, 223 individuals who are sensitive to the physical presence of others and social cues indicating 224 disapproval or judgement, would be ideally suited for online service delivery given the role it 225 plays in facilitating feelings of psychological safety and disinhibition (Leibert et al., 2006).

226 Moreover, due to the lack of verbal cues present in online service delivery, clients may 227 experience greater feelings of empowerment and autonomy over what they disclose to 228 practitioners, especially if it is sensitive information (Hanley, 2009).

In sum, given the perceived relative importance of SPPs having strong interpersonal skills

230 (Wooley \& Harwood, 2020), it is even more pertinent that practitioners pay careful attention to 231 the interpersonal skills they employ when consulting online, in an attempt to mitigate the 232 potential challenges of an absence of verbal and/or non-verbal cues when working online. 


\section{Technological Issues}

The use of technology in online service delivery has many associated benefits (e.g., accessibility, disinhibition, reflection) however, it is not without its limitations. In fact, technology has been identified as being a barrier to effective service delivery (Haberstroh et al.,

237 2007). Practitioners who use technology in the provision of online service delivery may experience internet connection issues or computer-related problems (Riemer-Reiss, 2000).

239 Subsequently, interference from technological issues may lead to both clients and practitioners 240 experiencing frustration, which could potentially thwart the working alliance (Haberstroh et al., 2412007 ; Haberstroh et al., 2008). If these technological issues persist or are unresolved this may

242 isolate a client for a prolonged period of time or in extreme cases, lead to the termination of an 243 intervention (Baker \& Ray, 2011). Therefore, in the event of any technological issues,

244 practitioners must have the basic technological competences to troubleshoot any software or 245 hardware issues and provide alternative means of service delivery (Riemer-Reiss, 2000).

\section{Concluding Remarks}

Given the growing use of technology and new media, practitioners have shown an increased interest in the role technology plays in service delivery. Despite this interest, there exists a limited sport psychology-specific knowledge base for professionals to draw from that

250 informs their practice when seeking to use media and technology in the provision of sport

251 psychology. Therefore, this presents scholars with a number of future research opportunities to 252 expand the evidence-base in which practitioners can draw from when consulting online. In particular, future research should aim to explore clients' and practitioners' perceptions of the

254 utility, ease of use, and effectiveness of technology in the online service delivery process.

255 Additionally, researchers should also seek to understand on how best to measure the effectiveness 256 of using technology and media in the provision of sport psychology. In this commentary we have attempted to draw to the readers' attention to several key opportunities and considerations relating to the use of technology in online psychological 
support. Namely, working alliance, accessibility, anonymity and disinhibition, time delays,

260 absence of verbal and nonverbal cues and, technological issues. As such, these opportunities and

261 considerations for using technology in online service delivery could act as a solid foundation in

262 which SPPs can draw on to inform their practice.

In conjunction with these opportunities and considerations, it must also be recognized that

264 online service delivery presents a range of ethical and legal implications that SPPs need to be

265 aware of and navigate. For example, SPPs need to consider confidentiality, competency, online

266 security, licensing and Gillick competence to name a few (Bird \& Harris, 2019; Cottrell et al.,

267 2018; Harris \& Birnhaum, 2015). Another key consideration that we acknowledge having

268 influence on the utility of working online, are the varying levels in which SPPs operate at,

269 notably one-to-one, dyadic, team and systemic levels (Sly et al., 2020). Therefore, in the next

270 section we offer some good practice recommendations for online service delivery at the

271 individual (e.g., athletes, coaches) and dyadic (e.g., athlete-coach, athlete-parent) level, which we

272 recognize may be less impactful at a systemic level.

\section{Recommendations of good practice}

274 - We recommend that practitioners undertake formal training in online service delivery to 275 develop core competencies and knowledge that are necessary when using technology in 276 the provision of sport psychology.

277 - The client's technological literacy ability, knowledge and confidence in using CMC

278 technologies should be assessed before introducing into the service delivery process.

279 - When working with children or young people, practitioners must assess whether the client 280 is Gillick competent before any consultancy can take place.

281 - Practitioners must also gauge whether using technology in the provision of sport 282 psychology will either aid or hinder the establishment, development and maintenance of 283 the working alliance. 
- Expectations regarding response times to messages (e.g., emails) should be clarified with the client at the start of the consultancy process, in order to guard against any anxieties that may arise from a delayed response.

- We recommend practitioners use noise cancelling headphones, to ensure confidentiality and eliminate any potential distractions that may occur outside the consulting room.

- Practitioners should carefully consider the background displayed when consulting online and where possible, use neutral backgrounds to avoid showing personal space which may result in discomfort being experienced by the client and/or practitioner.

- In the event of a crisis situation, practitioners should have a list of emergency contact details to hand, so they are able to coordinate emergency support in a timely manner.

- We also recommended that practitioners have a contingency plan for alternative service delivery approaches that mitigate against the potential negative consequences that may arise from any technological issues encountered.

- Webcams or in-built laptop cameras should be placed at eye level to avoid any perceived power imbalances by the client (e.g., practitioner looking down on client).

- Video chat-based software's (e.g., Zoom) often include a screenshare feature that is useful in making sessions interactive and offers a creative alternative to a whiteboard.

- Due to the potential absence or reduction of verbal and non-verbal cues available, practitioners should pay close attention to the cues available, in an attempt to understand the client's thoughts, feeling and emotions.

- Practitioners using technology are encouraged to adopt a flexible approach that allows them to alternate between technological and traditionally consultancy approaches, in order to meet the needs and expectations of the client.

- Given the labor intensive nature of online service delivery, we recommend practitioners 


\section{References}

311 Baker, K. D., \& Ray, M. (2011). Online counseling: The good, the bad, and the possibilities. Counselling Psychology Quarterly, 24(4), 341-346. https://doi.org/10.1080/09515070.2011.632875

314 Barak, A., \& Grohol, J. M. (2011). Current and future trends in internet-supported mental health interventions. Journal of Technology in Human Services, 29(3), 155-196. https://doi.org/10.1080/15228835.2011.616939

Barnett, J. E. (2005). Online counseling: New entity, new challenges. The Counseling Psychologist, 33(6), 872-880. https://doi.org/10.1177\%2F0011000005279961

Barazzone, N., Cavanagh, K., \& Richards, D. A. (2012). Computerized cognitive behavioural therapy and the therapeutic alliance: a qualitative enquiry. British Journal of Clinical Psychology, 51(4), 396-417. https://doi.org/10.1111/j.2044-8260.2012.02035.x

Bird, M. D., \& Harris, B. S. (2019). The ethical use of technology for clinical and performance enhancement services: Prevalence and perceptions among association for applied sport psychology certified consultants. Journal of Clinical Sport Psychology, 13(1), 56-71. https://doi.org/10.1123/jcsp.2017-0034

Blind Manuscript. Redacted authors (under review). The development of a novel method to support sustainable high-performance coaching. Journal of Applied Sport Psychology.

Bordin, E. S. (1979). The generalizability of the psychoanalytic concept of the working alliance. Psychotherapy: Theory, Research \& Practice, 16(3), 252-260.

Buffini, K. B., \& Gordon, M. (2015). One-to-one support for crisis intervention using online synchronous instant messaging: evaluating working alliance and client satisfaction. British Journal of Guidance \& Counselling, 43(1), 105-116. https://doi.org/10.1080/03069885.2014.987723 
ONLINE SERVICE DELIVERY

335 Chang, T. (2005). Online counseling: Prioritizing psychoeducation, self-help, and mutual help for counseling psychology research and practice. The Counseling Psychologist, 33(6), 881890. https://doi.org/10.1177\%2F0011000005279962

Cipolletta, S., Frassoni, E., \& Faccio, E. (2018). Construing a therapeutic relationship online: An analysis of videoconference sessions. Clinical Psychologist, 22(2), 220-229. https://doi.org/10.1111/cp.12117

Cotterill, S. T., \& Symes, R. (2014). Integrating social media and new technologies into your practice as a sport psychology consultant. Sport \& Exercise Psychology Review, 10(1), 55-64.

Cottrell, C., McMillen, N., \& Harris, B. S. (2019). Sport psychology in a virtual world: Considerations for practitioners working in eSports. Journal of Sport Psychology in Action, 10(2), 73-81. https://doi.org/10.1080/21520704.2018.1518280

Efstathiou, G. (2009). Students' psychological web consulting: Function and outcome evaluation. British Journal of Guidance \& Counselling, 37(3), 243-255. https://doi.org/10.1080/03069880902956983

Elleven, R. K., \& Allen, J. (2004). Applying technology to online counseling: Suggestions for the beginning e-therapist. Journal of Instructional Psychology, 31, 223-226.

Fletcher, D., \& Wagstaff, C. R. (2009). Organizational psychology in elite sport: Its emergence, application and future. Psychology of sport and exercise, 10(4), 427-434. https://doi.org/10.1016/j.psychsport.2009.03.009

Gelso, C. J., \& Hayes, J. A. (1998). The psychotherapy relationship: Theory, research, and practice. John Wiley \& Sons.

Haberstroh, S., Duffey, T., Evans, M., Gee, R., \& Trepal, H. (2007). The experience of online counseling. Journal of Mental Health Counseling, 29(3), 269-282. https://doi.org/10.17744/mehc.29.3.j344651261w357v2 
Haberstroh, S., Parr, G., Bradley, L., Morgan-Fleming, B., \& Gee, R. (2008). Facilitating online counseling: Perspectives from counselors in training. Journal of Counseling \& Development, 86(4), 460-470. https://doi.org/10.1002/j.1556-6678.2008.tb00534.x

Hanley, T. (2009). The working alliance in online therapy with young people: Preliminary findings. British Journal of Guidance \& Counselling, 37(3), 257-269. https://doi.org/10.1080/03069880902956991

Harris, B., \& Birnbaum, R. (2015). Ethical and legal implications on the use of technology in counselling. Clinical Social Work Journal, 43(2), 133-141. https://doi.org/10.1007/s10615-014-0515-0

Leibert, T., Archer Jr, J., Munson, J., \& York, G. (2006). An exploratory study of client perceptions of internet counseling and the therapeutic alliance. Journal of Mental Health Counseling, 28(1), 69-83. https://doi.org/10.17744/mehc.28.1.f0h37djrw89nv6vb

Mallen, M. J., Vogel, D. L., Rochlen, A. B., \& Day, S. X. (2005). Online counseling: Reviewing the literature from a counseling psychology framework. The Counseling Psychologist, 33(6), 819-871. https://doi.org/10.1177\%2F0011000005278624

Orlinsky, D.E., Rønnestad, M. H., \& Willutzki, U. (2004) Fifty years of psychotherapy processoutcome research: Continuity and change. In M. J. Lambert (Ed.), Bergin and Garfied's handbook of psychotherapy and behavior change (5th ed., pp. 307 - 390). New York: Wiley.

Quartiroli, A., Knight, S. M., Etzel, E. F., \& Zakrajsek, R. A. (2019). Fostering and Sustaining Sport Psychology Professional Quality of Life: The Perspectives of Senior-Level, Experienced Sport Psychology Practitioners. The Sport Psychologist, 33(2), 148-158. https://doi.org/10.1123/tsp.2017-0140

Richards, D., \& Viganò, N. (2012). Online counseling. In Encyclopedia of cyber behavior (pp. 699-713). IGI Global. 
385

386

387

388

389

390

391

392

393

394

395

396

397

398

399

400

401

402

403

404

405

406

407

408

Richards, D., \& Viganó, N. (2013). Online counseling: A narrative and critical review of the literature. Journal of Clinical Psychology, 69(9), 994-1011. https://doi.org/10.1002/jclp.21974

Riemer-Reiss, M. L. (2000). Utilizing distance technology for mental health counseling. Journal of Mental Health Counseling, 22(3), 189-203.

Rochlen, A. B., Zack, J. S., \& Speyer, C. (2004). Online therapy: Review of relevant definitions, debates, and current empirical support. Journal of Clinical Psychology, 60(3), 269-283. https://doi.org/10.1002/jclp.10263

Sessa, V. I., Hansen, M. C., Prestridge, S., \& Kossler, M. E. (1999) Geographically dispersed teams: An annotated bibliography. Center for Creative Leadership.

Sly, D., Mellalieu, S. D., \& Wagstaff, C. R. (2020). "It's psychology Jim, but not as we know it!": The changing face of applied sport psychology. Sport, Exercise, and Performance Psychology, 9(1), 87-101. https://doi.org/10.1037/spy0000163

Suler, J. (2004). The online disinhibition effect. Cyberpsychology \& Behavior, 7(3), 321-326. https://doi.org/10.1089/1094931041291295

Suler, J. (2010). Interpersonal guidelines for texting. International Journal of Applied Psychoanalytic Studies, 7(4), 358-361. https://doi.org/10.1002/aps.268

Thelwell, R. C., Wood, J., Harwood, C., Woolway, T., \& Van Raalte, J. L. (2018). The role, benefits and selection of sport psychology consultants: Perceptions of youth-sport coaches and parents. Psychology of Sport and Exercise, 35, 131-142. https://doi.org/10.1016/j.psychsport.2017.12.001

Woolway, T., \& Harwood, C. G. (2019). Gatekeepers' experiences of hiring a sport psychologist: a phenomenological study. Journal of Applied Sport Psychology, 31(4), 474-493. https://doi.org/10.1080/10413200.2018.1484394 
409 Woolway, T., \& Harwood, C. G. (2020). Consultant characteristics in sport psychology service provision: A critical review and future research directions. International Journal of Sport and Exercise Psychology, 18(1), 46-63. https://doi.org/10.1080/1612197X.2018.1462230 